\title{
Applications of Direct Electron Detection to the EBSD Analysis of Energy Conversion and Storage Materials
}

Matthew Nowell ${ }^{1}$, Rene de Kloe $^{2}$ and Stuart Wright ${ }^{3}$

${ }^{1}$ EDAX, Riverton, Utah, United States, ${ }^{2}$ EDAX, Tilburg, Noord-Brabant, Netherlands, ${ }^{3}$ EDAX, St Geprge, Utah, United States

Electron Backscatter Diffraction (EBSD) is a characterization tool that measures the crystallographic orientation and phase from an area of interest and uses this information to efficiently visualize and describe the microstructure, including characteristics of grain morphology, grain boundary character, texture, and localized misorientations. Traditionally EBSD patterns have been collected using CCD or CMOS-based cameras coupled to a phosphor screen which converted the backscattered electrons into light photons. Recently direct electron detectors have been used for EBSD collection [1]. These detectors eliminate the phosphor screen and optical components within the detection chain and improve pattern quality and detector sensitivity. This sensitivity is significant at lower beam energies and currents when analyzing beamsensitive materials.

EBSD has been used successfully to explore the role of microstructure on the performance of silicon, CdTe, and CIGS-based photovoltaic materials. Recent significant improvements in the performance of hybrid perovskite cells have driven interest in these materials' characterization [2-3]. These organic-inorganic materials present a challenge for EBSD characterization, as these materials are sensitive to damage from the electron beam at traditional beam doses. Direct electron detection technology allows for the efficient collection of EBSD data at milder analytical conditions. In this work, results from different methylammonium lead halide films will be presented, and the role of acquisition parameters discussed.

The demand for high-performance lithium-ion batteries is also increasing, with the most common cathode material being layered LiNixMnyCozO2 (NMC). The NMC particles are typically polycrystalline, and EBSD can resolve the internal microstructure. Characterization of the texture, grain size, and grain boundary structure can help understand their effects on performance [4-5]. However, the microstructure's spatial scale can require lower beam acceleration voltages to reduce the interaction volume within the NMC material to resolve the grain structure. Sample preparation of EBSD is key to quality results, and preparing energy conversion and storage materials for EBSD analysis can be challenging. Traditional approaches often fall short of the requirements of these materials. Broad ion beam preparation is a technique that produces a high-quality and representative surface for analysis. This approach can also be used on samples sensitive to air exposure to prevent sample degradation.

\section{References}

[1] A.J. Wilkinson, et al., Physical Review Letters 111 (2013) 065506.

[2] S. Jariwala, et.al., Joule 3 (2019) 1-13.

[3] G. Adhyaksa, et al., Advanced Materials 30 (2018) 1804792.

[4] A. Quinn, et al., Cell Reports Physical Science 1 (2020) 100137.

[5] Jing Li, et al. J. Electrochem. Soc. 164 (2017) A1534. 